\title{
Rethinking the proximodistal axis of the vertebrate limb in the molecular era
}

\author{
Cliff Tabin ${ }^{1,3}$ and Lewis Wolpert ${ }^{2,4}$ \\ ${ }^{1}$ Department of Genetics, Harvard Medical School, Boston, Massachusetts 02115, USA; ${ }^{2}$ Department of Anatomy and \\ Developmental Biology, University College, London WC1E 6BT, United Kingdom
}

The development of the vertebrate limb has long served as a paradigm for understanding the fundamental processes by which an undifferentiated field of cells gains spatial pattern and undergoes coordinated differentiation to produce the exquisitely complex structures that characterize our functional anatomy. The limb bud emerges from the flank as a mound of seemingly homogeneous mesenchymal cells within an ectodermal jacket. Yet within days this mass of cells gives rise to skeletal elements, muscles, and connective tissues organized in the form of a mature limb. Looking at one's own arm or leg, this organization can immediately be appreciated along three different axes: The back of the hand (dorsal) is different from the palm (ventral), the thumb (anterior) is distinct from the little finger (posterior), and the upper arm (proximal) is different from the lower arm and the hand (distal). The process by which differences along the proximodistal axis are established has been particularly controversial, with competing models proposed to explain the outcome of both classical and genetic manipulations. However, if one examines the two major models that have been previously proposed, neither of them is tenable in the context of our current knowledge of gene activity in the developing limb. Proximodistal patterning therefore needs to be placed into a new framework based directly on the molecular data.

The first key insight into the process of limb patterning came almost $60 \mathrm{yr}$ ago when John Saunders discovered that the apical ectodermal ridge (AER), a thickened ridge of ectodermal cells that runs along the anteriorposterior axis of the distal limb bud (equivalent to a ridge running along the distal edge of the hand from which the finger tips will eventually form) is necessary for the successful outgrowth of the limb along the proximodistal axis (Saunders 1948). To explain how different structures arise at different proximodistal levels as the limb bud grows out, a model was subsequently proposed based on progressive specification of increasingly distal cell fates in a domain $300 \mu \mathrm{m}$ deep directly below the AER (Summerbell et al. 1973). According to this view, under the

Correspondence.

${ }^{3}$ E-MAIL tabin@genetics.med.harvard.edu; FAX (617) 432-7595.

${ }^{4}$ E-MAIL 1.wolpert@ucl.ac.uk; FAX 44-207-813-2813.

Article is online at http://www.genesdev.org/cgi/doi/10.1101/gad.1547407. influence of the AER, cells are maintained in a so-called progress zone. These cells continuously acquire ever more distal positional information through the influence of an internal clock that is kept active as long as the cells receive signaling from the overlying AER. This process gives the cells a distal fate proportional to the length of time the cells remain in the progress zone. When cells move out of the range of AER signaling-i.e., out of the progress zone-the clock stops and their proximodistal fate therefore becomes fixed. This happens continuously, as all the cells in the progress zone-indeed, all of the cells of the limb bud-are dividing. As more cells are produced, only the members of the population closest to the distal end remain within the 300-um signaling range of the AER. Thus the first cells to be forced outside the progress zone, through the growth of the limb, become the upper arm while the last become the digits (Fig. 1A). (Note that the 300- $\mu \mathrm{m}$ depth of the progress zone is only an approximation, as are the sizes of several subsequently discussed regions such as the $200-\mu \mathrm{m}$ domain of cell death following AER extirpation. The numbers quoted are based on published estimates and give a rough indication of where in the limb bud certain events occur, but should not be considered to be precise measurements.)

This model can explain, in an elegant manner, several important classical experiments. First, the earlier in limb development the AER is removed, the more severe the truncation that results (Summerbell 1974; Rowe and Fallon 1982). Following early AER extirpation only proximal parts of the limb develop, while at later stages more distal structures additionally form. This can be interpreted as the consequence of prematurely stopping the clock within the cells of the progress zone upon AER removal. Cells lying within the progress zone would still contribute to limb structures but could only form them to the proximodistal level specified at the time of the surgery.

Second, when a subset of cells in the early progress zone are killed, for example, by $\mathrm{X}$ irradiation, distal structures form while proximal ones are reduced or absent (Goff 1962; Pinot 1970; Summerbell 1978; Wolpert et al. 1979). This is explained by the requirement that the depleted cell population in the progress zone needs to undergo additional proliferation before achieving a size 
A
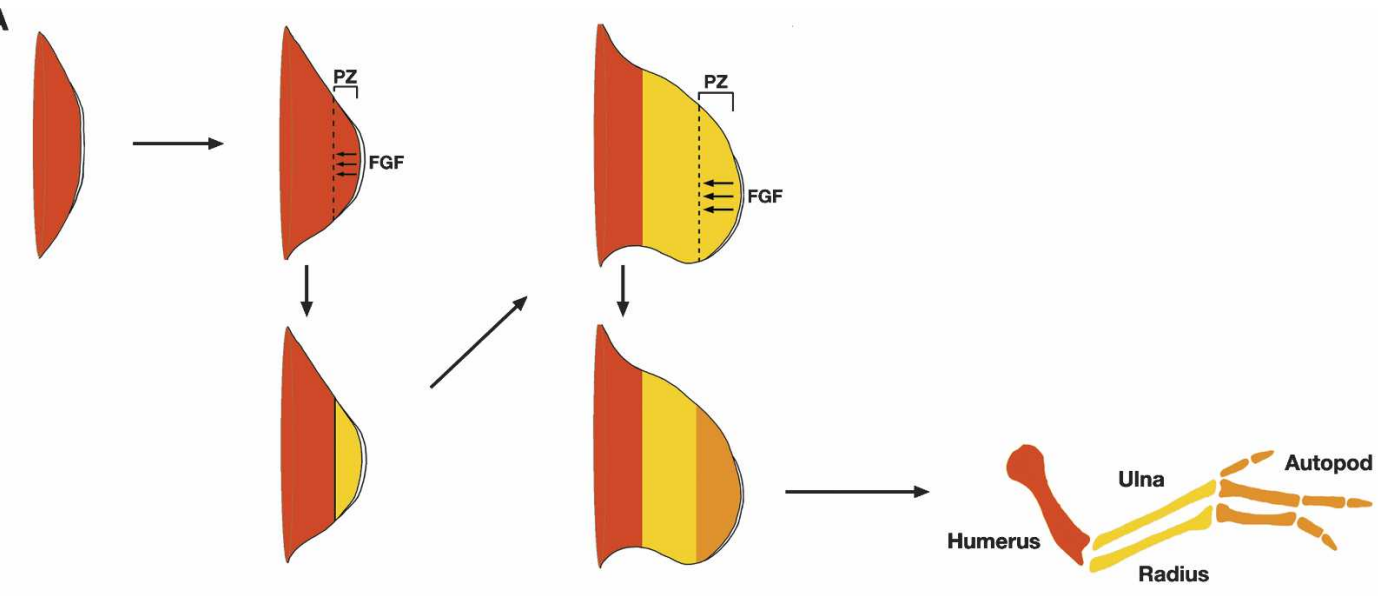

Progress Zone

Model

B

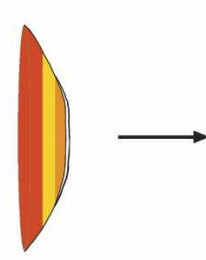

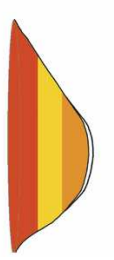
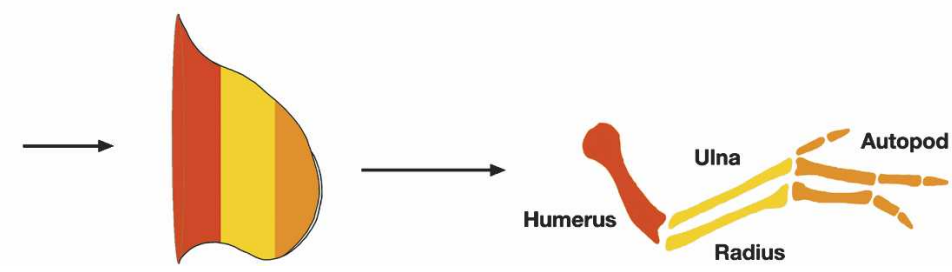

Early Specification Model

Figure 1. (A) The Progress Zone Model. Initially, the entire limb mesenchyme has a proximal identity. Left on its own it would develop into proximal, stylopod structures (red). However, the mesenchyme at the tip of the limb bud, the progreess zone (PZ), is exposed to Fgf and becomes respecified at a slightly more distal, zeugopod (yellow) fate. As limb development proceeds, the progress zone cells divide, and as a result of this growth, not all of the cells remain within range of the Fgf signal. Those too far from the tip maintain their already specified fate, whereas those still in close proximity to the tip are once again respecified to a still more distal, stylopod (orange) fate. $(B)$ The Early Specification Model. All of the proximodistal segments—stylopod (red), zeugopod (yellow), and autopod (orange) — are specified early in limb development. These segment-specific progenitor pools expand as the limb bud grows.

sufficient to force some cells out of reach of the AER. Until this happens, all the cells in the progress zone become distalized without leaving any outside the progress zone with a memory of more proximal fate specification.

Swapping AERs between leg and wing buds (Zwilling 1959) and between early and late stage limb buds (Rubin and Saunders 1972) has no effect on skeletal pattern. These results establish that the AER, while required for proximodistal outgrowth, does not instruct different proximodistal cell fates, and further indicate that the necessary factor(s) produced by the AER are the same for the wing and the leg and do not change with time. Subsequently, it was discovered that distal skeletal elements were rescued following AER extirpation by proteins of the fibroblast growth factor (Fgf) family (Niswander et al. 1993; Fallon et al. 1994) and at least four family members-Fgf4, Fgf8, Fgf9, and Fgf17-are indeed specifically expressed in the mouse AER (Martin 1998; Sun et al. 2000). Thus the progress zone could in principle be redefined as the set of cells within the distal limb bud that are within range of Fgf signaling from the AER. According to this view, Fgfs are the permissive factors that allow the progress zone cells to maintain the activity of their internal clock and hence permit their continued, progressive acquisition of ever more distal fates.

More recently, an alternative view that can be termed the "Early Specification Model" was developed to ex- plain the distal truncation seen following AER removal (Dudley et al. 2000). Extensive apoptosis is seen in the distal-most $200 \mu \mathrm{m}$ following AER extirpation (Rowe et al. 1982; Dudley et al. 2002). As the limb grows, the 200- $\mu \mathrm{m}$ domain of cell death becomes proportionally a smaller portion of the total limb bud, leading to progressively less distal truncations in skeletal pattern. Indeed, fate mapping indicates that marked distal cells are not incorporated into skeletal structures following AER removal (Dudley et al. 2002). Thus, if the early specification model were correct, the skeletal truncations following AER extirpation would reflect the apoptotic loss of already specified distal segments (rather than indicating the extent of distal specification at the time of the experiment, as the Progress Zone Model would propose).

Both the Progress Zone Model and the Early Specification Model can explain the classical AER extirpation and X-irradiation experiments (for review, see Mariani and Martin 2003). Nonetheless, when critically assessed in light of more recent molecular data, neither of these models is completely satisfactory. The phenotypes resulting from conditional removal of $\mathrm{FgF}$ gene activity in the AER (Lewandoski et al. 2000; Sun et al. 2002) are difficult to reconcile with the Progress Zone Model without some additional less-than-straightforward assumptions. Moreover, there is currently no convincing evidence for a cell-autonomous clock capable of affecting 
limb patterning, which is at the heart of the Progress Zone Model. It is worth noting, in this regard, that very intriguing cycles of expression of the gene hairy2 have recently been identified during distal limb outgrowth (Pascoal et al. 2007). However as hairy2 is believed to function to transduce Notch signaling, and genetic removal of all canonical or noncanonical Notch activity in the limb fails to produce a segmentation phenotype (Pan et al. 2005), the relevance of this observation remains unclear. Thus, while it is certainly an exciting finding worthy of additional study, for the moment at least a concrete molecular mechanism for a progress zone clock is still lacking. On the other hand, the Early Specification Model does not provide an explanation for how the distinct proximodistal progenitor pools are established, nor is it clear whether there is even space in the earliest limb bud to accommodate distinct zones of cells corresponding to each of the later limb segments (Wolpert 2002). In our view, however, the biggest problem with both of these models is the rather striking lack of support for either one from molecular expression studies.

The Progress Zone Model makes a strong prediction that the entire population of progress zone cells, $300 \mu \mathrm{m}$ wide, would coordinately undergo successive changes in the expression of a series of distinct specification genes. Moreover, according to that model, the changes in proximodistal specification gene expression would be based entirely on an internal clock and would not be dependent on inductive signaling. The Early Specification Model makes the equally strong prediction that the expression of specification genes would form small stripes across the early limb bud that would subsequently expand into wider stripes as the respective progenitor pools proliferate. It has been 17 years since the nested patterns of Hox genes provided the first molecular insights into limb patterning (Dolle et al. 1989), and literally hundreds of limb expression patterns have since been described, including markers for each of the developing proximodistal limb segments. Yet no patterns have emerged such as those predicted by either of the two major models of proximodistal patterning. There are no genes known to arise progressively in the distal mesenchyme that are independent of instructive inductive cues, and there are no genes known to be expressed in stripes reflective of the future limb segments in the earliest limb bud. Indeed, the best markers for the individual limb segments, such as Hoxa13 for the autopod, are expressed in patterns that directly contradict both models (see below).

One can always argue that true proximodistal specification genes, with expression patterns as predicted by one of the two models, remain undiscovered. However, eventually such an excuse for the lack of evidence begins to ring hollow, especially as several large-scale expression screens by in situ hybridization and microarray have not revealed genes with patterns matching the predictions of either of the two models. A parallel can be drawn to the state of paleontology in the late 1970s when the failure to observe gradual changes in the fossil records could no longer satisfactorily be excused on the basis of an incomplete fossil record, and punctuated equilibrium was finally appreciated as a major mode of evolutionary change (Gould and Eldredge 1972). We have, therefore, re-examined the available molecular information on gene expression along the proximodistal limb axis to see if the existing data itself could form the basis for a new way of conceptualizing proximodistal specification.

\section{Toward a modern view of proximodistal specification}

A gene activity-based framework for understanding the specification of the most proximal portion of the limb (the stylopod) has already been provided by Mercader et al. (2000). They describe the critical role of two signaling centers in establishing this aspect of proximodistal patterning, one in the flank (producing retinoic acid) and one at the distal tip of the limb bud (producing members of the Fgf family). We expand on this model to additionally address the specification of more distal limb segments by considering the dynamic changes in gene expression in the distal limb and the signaling centers that coordinate them. In addition, we consider another key level of complexity in the process of defining the limb segments. Even as the progenitors of the eventual proximodistal segments are being specified, the progenitor pools that will ultimately contribute to each limb segment are rapidly expanding through continued proliferation. We suggest that the length of time cells proliferate before committing to differentiation will significantly influence the size of the progenitor pools at the time differentiation is initiated, which consequently will have a major impact on the ultimate size of the skeletal elements produced. Thus, although the humerus and phalanges are normally of similar size when they first condense, if the humerus progenitors proliferate for a shorter period of time before commencing differentiation, the humerus condensation and hence the element itself will be shorter than normal.

The cells at the distal tip of the limb bud are maintained in an apparently undifferentiated state due to the influence of signals from the AER /Globus and Vethamany-Globus 1976). To put this observation in modern terms, we suggest that during limb bud outgrowth, commitment to differentiation and chondrogenic condensation occurs as cells exit the domain influenced by Fgf signaling from the AER. We will refer to this Fgf-responding domain that roughly corresponds to the distal region previously identified as the progress zone, as the "undifferentiated zone." We will refer to the proximal border of this domain as the "differentiation front." Initially the undifferentiated zone encompasses the entire limb bud, but as the mass of mesenchyme becomes large enough, the most proximal cells are not longer within the range of Fgf signaling and, therefore, are able to enter various differentiation pathways. This process continues with the resultant well-known wave of proximal-to-distal differentiation (Fig. 2A). As this is occurring, dynamic patterns of gene expression are unfolding. Without reference, for now, to the way proximodistal gene expression is regulated, we postulate that a cell's proximodistal 


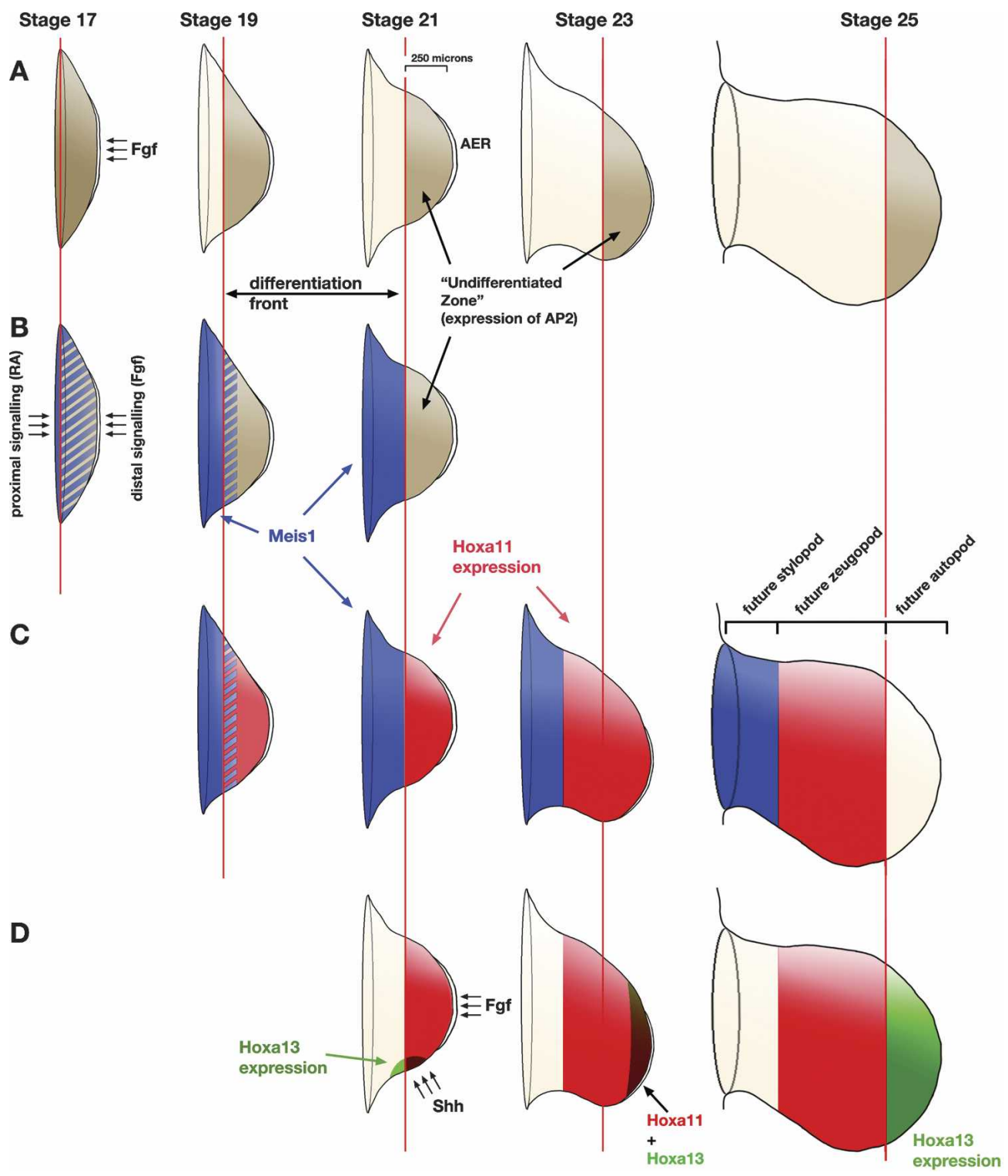

Figure 2. Establishment of distinct molecular domains along the proximodistal limb axis. (A) Approximate map of the undifferentiated zone in the distal $250 \mu \mathrm{m}$ of the limb bud $(\tan )$ at various stages of limb development. (B) Comparison of the domains of expression of Meis1 (a stylopod marker; blue) and AP2 (an undifferentiated zone marker; tan) at different stages of limb development. (C) Comparison of the domains of expression of Meis1 (a stylopod marker; blue) and Hoxa11 (a zeugopod marker; red) at different stages of limb development. $(D)$ Comparison of the domains of expression of Hoxal1 (a zeugopod marker; red) and Hoxal3 (an autopod marker; green) at different stages of limb development. Hoxa11 and Hoxa13 expression overlap at early stages of their expression (brown).

specification is based on the segment-specific genes it expresses at the time it exits the undifferentiated zone.

While the mechanisms that pattern the distinct limb segments remain controversial, we can follow the evolving domains of segment-specific gene expression as a surrogate providing an indication, in relative terms, of when the limb segments are specified and gain insight from the way those genes are regulated. On such a basis, the first segment of the limb to be specified in the model we are proposing is the stylopod, under the influence of the proximal and distal signaling centers as suggested by
Mercader et al. (2000) (Fig. 2B). Because these signaling centers are fairly close to one another in early, tiny limb buds, the expression domains of genes positively regulated by each of these signaling centers initially overlap. As the limb bud grows and the distance between the proximal and distal signaling centers increases and the expression domains of the genes they regulate became distinct (Fig. 2B).

Thus, as the early limb bud starts to expand, the most proximal cells are the first to find themselves out of range of the AER. Because they are beyond the influence 
of AER signals, but are close to the source of flank-derived signals, the moment they cross the differentiation front they cease expressing distal markers and only maintain stylopod-specific gene expression. At the same time, as they are pushed out of range of the AER signals, they commit to enter cellular differentiation pathways. This process continues as the limb bud grows, with more progenitors escaping the influence of the AER and joining the stylopod until the limb bud is large enough that cells forced by cell expansion out of range of the AER are also out of range of flank signals. At that point, we suggest that the size of the stylopod progenitor pool has been fixed, and subsequent cells pushed across the differentiation front will form more distal limb segments.

In our aquatic ancestors, this might have been all that was required for proximodistal specification, as the autopod (wrist/ankle and digits) is believed to be a "neomorphic" evolutionary innovation of the tetrapod lineage (Sordino et al. 1995) and the ancestral appendage was in essence limited to a stylopod and a zeugopod. In that setting, the proximal and distal signaling centers would be sufficient to establish two proximodistal domains, one established within the range of the flank signals and one out of range of the flank signals.

In tetrapods, where a series of distal limb segments needs to be specified and expanded, the process becomes more complex. As the entire limb bud distal to the stylopod is out of range of flank signaling, this proximal signaling center ceases to be important for more distal specification. However, a third signaling center, the zone of polarizing activity (ZPA) acts in concert with the AER to initiate dynamic patterns of expression that evolve through cross-regulation within the distal cells themselves as well as under the continuing influence of the AER and ZPA.

Of course, the ZPA is traditionally thought of as a signaling center organizing the anteroposterior axis. However, the reality is that limb patterning is achieved as an integrated whole, with critical cross-interactions between the signaling centers patterning the several limb axes as well as integration at the level of the regulation of key target genes, such as the Hox genes (see below). In particular for our current discussion, we will argue that the activity of the ZPA is also important to take into account in considering the regulation of genes specifically expressed in the zeugopod and autopod.

How we view signals from the AER and ZPA influencing the proximodistal patterning of the zeugopod and autopod can be more easily explained in the context of the gene expression patterns described below. As in the specification of the stylopod, it will be seen that there is a coordination between the dynamic changes in distal expression domains and the onset of differentiation, as the effective range of AER signaling demarcates both the subset of cells in the distal limb bud where proximodisal gene expression patterns can be modified by continued AER signaling and the depth of the undifferentiated zone.

For simplicity, in the discussion that follows we will focus exclusively on markers for the major subdivisions of the limb, the stylopod, zeugopod, and autopod. However, to fully understand proximodistal patterning, ultimately additional segments must also be considered. The wrist/ankle are distinct from digits within the autopod. Moreover, at least in principle, each phalanx within a digit could also be viewed as a distinct segment. In our discussion (below) of the molecular data in support of our model, we will continue to focus on markers for each of the three major limb subdivisions; for the sake of simplicity, however, it will be important in the future to expand this analysis, taking into account additional proximodistal domains. For example, specification of the wrist could be followed utilizing the difference in late expression patterns of Hoxa13 (entire autopod) and Hoxd13 (autopod exclusive of wrist).

\section{Markers for proximodistal limb segments}

The best markers available for the stylopod segment are Meis1 and Meis2, which encode related TALE-homeodomain proteins. The Meis proteins regulate the activities of the transcription factor Pbx and Hox genes (for review, see Moens and Selleri 2006). The Meis genes are more than just markers for the stylopod, as they may themselves play a role in proximal specification; since ectopic expression of these genes can reprogram proximodistal fate at least to some extent (Capdevila et al. 1999; Meracader 2000). However, it is important to note that gene inactivation studies have indicated that neither of these genes is essential, at least individually, for normal limb development (Azcoitia et al. 2005).

Arguably the best markers discovered thus far for the distal limb segments are the $5^{\prime}$ members of the Hoxa and Hoxd clusters, at least at later stages of development. Early in limb development, these genes have dynamic patterns of expression (see below); however, by the time cells in the chondrogenic condensations start to differentiate into cartilage, Hoxa11 is an excellent and very precise marker for the entire zeugopod, while Hoxal3 is an equally good marker for the entire autopod (Yokouchi et al. 1991). It is important, however, to bear in mind that these genes are themselves not actually involved in segment specification. For example, when all Hoxall activity is removed from the limb bud (Hoxall and Hoxd11 in the forelimb [Davis et al. 1995], and Hoxa11, Hoxc11, and Hoxd11 in the hindlimb [Wellik and Capecchi 2003]), the zeugopod condensations (the cartilaginous anlagen of the radius and ulna or tibia and fibula) form relatively normally. In the absence of the influence of these Hox genes, however, there is a failure to form normal growth plates at the proximal and distal ends of the zeugopod bones and the zeugopod is extremely reduced (Boulet and Capecchi 2004). Thus Hox11 gene activity is indeed critical, quite specifically for normal zeugopod development, but is not required to specify the zeugopod itself.

Although the Hoxa genes do not appear to play a role in segment specification per se, their final expression domains are exactly congruent with the distal limb segments. Thus, their regulation must reflect a mechanism 
for bringing their domains into precise register with the segment boundaries. Hence, either they are regulated by the true specification genes, or are coregulated by the same processes that control the expression of the specification genes. In either case, since no genes directly involved in distal specification have been described, these Hox genes can be used as surrogates for understanding the formation of the distinct zeugopod and autopod segments.

In addition to the genes marking the future limb segments, there are also genes whose expression reflects the activity of the signaling centers influencing the proximodistal axis. Thus the Retinoic Acid Receptor $\beta$ $(\mathrm{RAR} \beta)$ is expressed in the proximal-most limb reflecting retinoid signaling from the flank (Dolle et al. 1990; Smith and Eichele 1991). Similarly, a large number of genes expressed distally throughout limb development are thought to be directly regulated by Fgfs produced within the AER. It is worth noting, however, that for most of these genes the assumption that they are regulated by FGF signaling from the AER is based on AER removal experiments, in which the subridge cells die within a few hours of ridge removal. Thus the observed loss of expression of these genes and rescue of their expression following ridge removal by the application of FGF beads might be due to cell death and prevention of cell death, respectively, rather than any direct effect of FGF signaling on the genes themselves. Nevertheless, several of these genes are expressed in multiple places in the developing embryo in regions of Fgf signaling, supporting the idea that they could indeed be Fgf dependent in the distal limb as well. These genes-including, for example, AP2 (Chazaud et al. 1996; Shen et al. 1997), Fgf10 (Ohuchi et al. 1997), Sprouty1 and Sprouty2 (Chambers and Mason 1999; de Maxim et al. 1999; Minowada et al. 1999), and the distal mesenchymal domain of Msx1 (Ros et al. 1992)-are expressed to slightly differing distances below the AER, presumably reflecting distinct levels of response to a distal-to-proximal Fgf protein gradient. The most broadly expressed of these extends roughly $250 \mu \mathrm{m}$ from the distal tip. The size of the expression domain of any one of these genes is relatively constant through the patterning phase of limb development. In this respect, they are similar to the relatively constant-sized distal zone where the mesenchyme is kept in an apparent undifferentiated state by signaling from the AER (Globus and Methamany-Globus 1976). Thus, we consider expression of genes such as AP2 as markers for the distal region where cells are held in an undifferentiated state, rather than reflecting a particular state of proximodistal specification. We do not, however, mean to imply that any of these specific distal markers are themselves necessarily involved in regulating commitment to differentiation.

\section{Dynamic patterns of expression in the context of proximodistal specification}

For simplicity, we will focus on four genes as markers for this discussion: Meis1 for the stylopod, Hoxa11 for the zeugopod, Hoxa13 for the autopod, and AP2 for the un- differentiated zone actively responding to AER/Fgf signaling. When the limb bud first becomes evident /stage 17 in the chick forelimb), Meis1 is expressed throughout the limb bud (Capdevila et al. 1999; Mercader et al. 2000) presumably in response to signaling from the flank (Fig. 2B; Mercader et al. 2000). At the same time, the entire limb bud also expresses AP2 under the presumed influence of AER signals (Chazaud 1996; Shen 1997). As first discussed by Mercader et al. (2000), the complete overlap of these domains can fit with a model based on proximal and distal signaling centers, since at early stages, every cell in the limb bud should be within range of both flank and AER signaling, although direct evidence for the necessity of a proximal signaling center in limb development is lacking. As the limb bud grows, three different zones emerge, a proximal domain that continues to express Meis1, a distal domain expressing only AP2, and a middle region expression both genes. These observations also fit the model, since it predicts that as the limb bud enlarges, cells in the proximal region will only be within range of signals from the flank, cells in the distal region will only be within range of signals from the AER, and cells in the middle region will be within range of both signals. At still later stages, the Meis1-expressing domain proximally and AP2-expressing domain distally are nonoverlapping, and a domain of increasing size is generated between them where neither of these markers is expressed. Again, this fits the model, which predicts that at later stages there will be a domain in which cells are out of range of both flank and AER signals.

Against the backdrop of the expansion and separation of the Meis1 and AP2 expression domains, Hoxa11 and Hoxa13 are activated, and their expression patterns evolve to ultimately correspond to the zeugopod and autopod, respectively. Hoxal1 expression is initiated at a time when Meis1 and AP2 still overlap significantly (stage 19 in the chick forelimb) (Nelson et al. 1996; Vargesson et al. 2001), in a posteriorly based distal stripe overlapping both Meis1 and AP2 (Fig. 2C). Over time, however, the proximal domain of Hoxal 1 expression becomes restricted such that it attains a mutually exclusive domain relative to Meis1 (Capdevilla et al. 1999; Mercader et al. 2000). Thus, once Hoxa11 expression has been initiated in the distal limb overlapping the early domain of Meis1, like Mercader et al. (2000), we propose that the subsequent mutually exclusive pattern of expression of Meis1 and Hoxa11 is achieved through a combination of flank signals promoting Meis1 expression, distal signals antagonizing Meis1 expression, and mutual negative regulation between Meis1 and Hoxa11 enhancing the transition (see below). Note, however, that to the extent that flank signals play a role in establishing proximodistal limb boundaries, they must do so relatively early, as a mid-stage limb bud can be grafted to the dorsal surface of a second, host limb bud and a normal proximodistal skeletal pattern will result, in the absence of any continued interaction with flank signals. Thus from mid-bud stages onward the distal signals combined with mutual Meis1-Hoxa11 antagonism must suffice to refine their mutually exclusive domains. 
Since, in this model, the range of Fgf signaling defines both the territory where Meis 1 will be repressed and the extent of the distal undifferentiated zone, it assures that the Meis1 and Hoxa11 zones finally become mutually exclusive precisely at the time the last of the Meis1expressing cells are pushed across the differentiation front by the expansion of the limb bud. Subsequent cells to cross the differentiation front thus express Hoxa11 but not Meis1. Hence, in the early limb bud, proximal cells start differentiation while expressing only Meis1, and when subsequently the mutually exclusive domains of Meis 1 and Hoxa11 are established, cells start differentially expressing only Hoxa11. In other words, while cells transiently express both these markers in the early limb bud, the coordination of their dynamic expression with the differentiation front means that at the time differentiation actually takes place cells express either the stylopod or zeugopod marker, but not both.

Shortly after Hoxa11 expression is dissociated from Meis1 expression at the proximal limit of the Hoxa11 domain, Hoxa11 expression is down-regulated in the distal handplate such that it also has a mutually exclusive border with the late distal expression domain of Hoxa13. Hoxa13 expression is initiated after Hoxa11 (stage 21 in the chick forelimb) (Nelson et al. 1996; Vargesson et al. 2001), in a very small domain at the posterior-distal margin, initially overlapping with both Meis1 and Hoxa11 (Fig. 2D). Hoxa13 expression then expands to form a narrow distal stripe overlapping with the distal expression of Hoxal1 but no longer overlapping the proximal expression domains of Meis1 (stage 23 in the chick forelimb). Finally, Hoxa13 expression broadens to encompass the distal $200-300 \mu \mathrm{m}$ as the handplate extends (stage 25 in the chick), at the same time as Hoxall is down-regulated distally (Yokouchi et al. 1991; Nelson et al. 1996). Once again, we suggest that the process of distal Hoxa13 expansion and Hoxa11 down-regulation is coordinated with the time at which cells leave this undifferentiated zone such that cells do not cross the differentiation front while expressing both Hoxa11 and Hoxa13. At the same time as the Hoxa11 is completely down-regulated distally and the Hoxa13 expression domain reaches its full 200- to 300-um width, the last of the Hoxa11-expressing cells moves across the differentiation front. Subsequently, when differentiation is initiated in more distal cells, they express only Hoxa13. At this point, the three cardinal limb segments, the stylopod, zeugopod, and autopod, are each distinctly defined by the expression of Meis1, Hoxa11, and Hoxa13, respectively.

\section{Regulation of gene expression along the proximodistal axis}

Of these markers, it is easiest to understand the regulation of AP2, which is expressed in the distal undifferentiated zone mesenchyme at every stage of limb bud development. In all likelihood, AP2 (like other markers for this domain) is strictly dependent on Fgf signaling from the AER. Following AER extirpation, AP2 expression is completely lost within 4-8 h, and this down-regulation can be rescued by ectopic Fgf (Shen et al. 1997). Other distal makers have similarly been shown to be exquisitely sensitive to loss of the AER. For example, the mesenchymal expression of Msx1 subjacent to the AER is down-regulated as quickly as $2 \mathrm{~h}$ after AER removal and completely absent after $4 \mathrm{~h}$ (Ros et al. 1992). Like AP2, distal Msx1 expression can be maintained by ectopic application of Fgf-soaked beads (Fallon et al. 1994). Moreover, other distally expressed genes such as the markers of the Sprouty family are not only expressed in the domain of the distal Fgf signaling in the limb bud, but also in many other areas of Fgf signaling in the embryo. Thus, although in principle the expression of these genes in the distal limb bud could be independent of Fgf signaling, in which case (as discussed above), the down-regulation of AP2, Msx1, Sprouty, and other genes following AER removal could be attributed to the rapid wave of distal cell death that follows AER extirpation (Rowe et al. 1982; Dudley et al. 2002), the most parsimonious explanation is that they do depend on active Fgf signals in the limb. Moreover, even if those specific genes are not themselves regulated by Fgf, it is clear from the 200- to 250$\mu \mathrm{m}$ region of distal cell death following AER removal that their expression domains are approximately coincident with the range of Fgf signaling and can therefore be used as surrogates for following the cells responding to Fgf as the limb bud expands along the proximodistal axis.

Using Meis1 as a marker for the proximal limb segment, the entire early limb bud transiently carries this molecular signature of the stylopod. It has been postulated that the expression of Meis is based on signaling by retinoic acid (RA) from the flank, as ectopic RA expands Meis1 expression distally at later stages while RA antagonist prevents proximal Meis1 expression (Mercader et al. 2000). Interestingly, Meis 1 expression also appears to be negatively regulated by Fgf signaling, as the endogenous Meis1 expression is down-regulated by ectopic Fgf protein (Mercader et al. 2000). Thus one can hypothesize that, at the earliest stages when the entire limb bud presumably sees high levels of both RA and Fgf, RA in effect "wins," and as a consequence Meis 1 is expressed. As the limb bud grows, gradients of both molecules are presumably set up such that toward the middle of the limb bud, cells see lower RA concentrations, under which conditions Fgf is able to act as an inhibitor of Meis1, and in this way could contribute to the formation of the sharp boundary of Meis1 expression.

The regulation of Hoxa11, the marker of the zeugopod segment, is less clear. As discussed above, Hoxa11 expression is initiated in a broad distal domain, under the AER at a time when most of the limb bud also expresses Meis1. There are reports that, like the Hoxd genes (Laufer et al. 1994; Koyama et al. 1995; Vogel et al. 1995), Hoxa11 requires Fgf signaling from the AER (Hashimoto et al. 1999). However, this is only observed at early stages (stage 20 in the chick forelimb), whereas Hoxa11 expression is stably expressed and independent of AER activity at later stages (stage 25 in the chick forelimb) (Hashimoto et al. 1999; Vargesson et al. 2001). This 
could represent a transition in Hoxal1 regulation, but in light of the current appreciation of distal cell death following AER removal (Rowe et al. 1982; Dudley et al. 2000), an alternative explanation would be that Hoxa1 1 expression is never directly dependent on AER signaling. Rather, at early stages the relatively small and distally restricted domain of Hoxa11 expression is lost by apoptosis following AER removal. At later stages AER removal results in loss of distal cell populations, but the Hoxal1 domain is larger and extends more proximally than the wave of cell death and hence is largely unaffected by it. Consistent with this explanation for the loss of Hoxal1 in early limb buds, an Fgf bead can rescue Hoxa11 expression if applied immediately upon AER extirpation, but not $12 \mathrm{~h}$ later (Hashimoto et al. 1999), when the wave of apoptosis has already taken place (Dudley et al. 2000).

In contrast, Hoxa13 does seem to be directly regulated by Fgf signaling (Hashimoto et al. 1999; Vargesson et al. 2001). AER removal results in a very rapid loss of Hoxa13 expression, which is rescued by application of exogenous Fgf (Vargesson et al. 2001). However, ectopic Fgf is not capable of expanding the Hoxa13 domain proximally, suggesting that additional signals must also be involved in establishing the limits of its domain of expression (Vargesson et al. 2001). One such factor that could act to restrict Hoxa13 to its normal distal domain is RA, since ectopic application of RA results in a down-regulation of Hoxa13 (Mercader et al. 2000). Interestingly, when ectopic RA blocks distal Hoxa13 expression, there is a concomitant distal expansion of Hoxal1 expression. Thus the effect of RA on Hoxa11 appears to be position dependent. At the proximal border of its normal domain, RA signaling inhibits Hoxa11 expression, whereas at its distal border, RA induces Hoxa11. This can be interpreted in terms of reciprocal and mutually antagonistic proximodistal gradients of RA and Fgf (Mercader et al. 2000).

Not surprisingly, there are also regulatory relationships between these various transcription factors themselves. Consistent with their mutually exclusive domains of expression, Hoxa13 misexpression represses Meis1 while Meis1 misexpression down-regulates Hoxa13 (Capdevila et al. 1999). Test for cross-interactions between Meis1 and Hoxal1 have not been reported, but it seems likely that these genes also have a reciprocal negative regulatory relationship. Perhaps surprisingly, however, Hoxal1 and Hoxa13, which share a very sharp boundary in the late limb bud, do not appear to directly regulate one another, and Hoxa13 misexpression has no effect on Hoxal1 expression (Yokouchi et al. 1995). This suggests that perhaps their adjacent, nonoverlapping expression domains are established by reciprocal response to a common signal.

One possible candidate for a common signal affecting both Hoxa11 and Hoxa13 expression is Sonic hedgehog (Shh). While Shh acts mainly along the anterior-posterior axis, its activity is integrated at multiple levels with signals primarily affecting other axes. For example, Shh is known to be required in addition to Fgf signals for Hoxd13 expression (Koyama 1993; Laufer 1994). Hoxa13, described above, is initiated in a tiny posterior-distal domain that correlates with the location of highest Shh signaling in the ZPA. Moreover, recent studies of Hoxa13 and Hoxa11 expression in Shh-deficient mouse embryos shows that Hoxa13 expression is lost in the absence of Shh, and at least in preliminary analyses, Hoxa11 fails to be down-regulated in the distal limb bud (T. Toyoaki and A. McMahon, pers. comm.).

In short, these collective studies reveal a complex set of regulatory interactions controlling the temporally dynamic expression patterns of AP2, Meis1, Hoxa11, and Hoxa13. Ultimately these patterns resolve in final expression domains that uniquely define each of the future limb segments. We have suggested that establishing these domains of gene expression depends on both inductive signaling and cross-regulation between the genes themselves. The coordination of these events with the expansion of cells across the differentiation front assures that cells only express genes characteristic of a single limb segment at the time they start differentiation. We would further propose that when a discontinuity between progenitor pools is reached-for example, when Hoxa13-positive cells follow Hoxal1-positive cells across the differentiation front-they will have distinct cellular properties and respond differently to extracellular cues, leading to the initiation of a new limb segment and distinct skeletal elements.

Through expression analysis of Meis1, Hoxa11, and Hoxa13 at progressive stages of limb development, it becomes apparent that they do not support either the Early Specification or Progress Zone Models of proximodistal specification. The expression domains of these markers are very dynamic. At early stages they are not found in a simple prepattern of the limb segments that simply expands, as would be suggested by the Early Specification Model. Thus, the molecular data suggest that the Early Specification Model simply cannot be right. Similarly, the activation of Hoxa13 in a tiny posterior-distal domain that expands first into a narrow distal stripe at the distal tip (much thinner than the postulated progress zone) and then spreads into a 250 - $\mu \mathrm{m}$-wide handplate does not match the coordinated promotion of all the cells within a 250 - $\mathrm{um}$ progress zone to an autopod fate, as is hypothesized by the Progress Zone Model. Moreover, the regulation of the genes considered here depends on extracellular induction of gene expression rather than being established by an internal, autonomous clock, the mechanism that is at the heart of the Progress Zone Model. While one can, nonetheless, hypothesize that eventually additional markers will be identified whose expression patterns and modes of regulation will support either the Early Specification or the Progess Zone Model, we suggest that until such genes are indeed found, it will be most productive to base thinking about the proximodistal limb axis on the data that do exist.

In summary, neither the Progress Zone Model nor the Early Specification Model fit the large amount of molecular expression data generated in the last decade. As an alternative, we have utilized these data to provide a new framework for viewing proximodistal lamb pattern- 
ing. The emerging picture is one of dynamic expression domains of specification genes in the context of the growth of the limb bud and a distally maintained differentiation front. The result is the progressive generation and expansion of progenitor pools specific for each limb segment. This is not so much a new model as a dynamic description of molecular events responsible for defining proximodistal limb segments.

A major weakness in the foregoing discussion is that the genes we use as markers for proximodistal specification in our analysis are, with the possible exception of Meis1, not actually involved in determining segment identity. They are, however, the best markers currently available, and (as discussed above) are brought into quite precise register with the segment primordial prior to the overt differentiation of the proximodistal elements. Even so, it remains possible that the true proximodistal specification genes are regulated quite differently. Nonetheless, we have undertaken this survey of currently known gene regulation out of the belief that the current molecular data demand a shift in the way we think about proximodistal patterning, and the importance of this analysis, if any, lies in a way of viewing the problem rather than in the details. It is important that specification of the proximodistal axis be conceptualized on a molecular basis and that future molecular and genetic studies be focused in that direction rather than toward proving or disproving the classical models. At the same time, it will be extremely important to revisit the classical experiments, such as AER extirpation and X-irradiation studies, with modern molecular tools to gain a deeper understanding of proximodistal limb patterning and how it is established.

\section{Acknowledgments}

We gratefully thank Gail Martin, Francesca Mariani, John Fallon, and members of the Tabin laboratory for many helpful and detailed comments on drafts of this essay. Work on limb patterning in the laboratory of C.T. is supported by a grant, HD032442, from the NIH.

\section{References}

Azcoitia, V., Araci, L.M., Martinez-A, C., and Torres, M. 2005. The homeodomain protein Meis1 is essential for definitive hematopoiesis and vascular patterning in the mouse embryo. Dev. Biol. 280: 307-320.

Barna, M., Pandolfi, P.P., and Niswander, L. 2005. Gli3 and Plzf cooperate in proximal limb patterning at early stages of limb development. Nature 436: 277-281.

Boulet, A.M. and Capecchi, M.R. 2004. Multiple roles of Hoxal1 and Hoxd11 in the formation of the mammalian forelimb zeugopod. Development 131: 299-309.

Capdevila, J., Tsukui, T., Rodriquez Esteban, C., Zappavigna, V., and Izpisua Belmonte, J.C. 1999. Control of vertebrate limb outgrowth by the proximal factor Meis2 and distal antagonism of BMPs by Gremlin. Mol. Cell 4: 839-849.

Chambers, D. and Mason, I. 2000. Expression of sprouty2 during early development of the chick embryo is coincident with known sites of FGF signalling. Mech. Dev. 91: 361-364.
Chazaud, C., Oulad-Abdelghani, M., Bouillet, P., Decimo, D., Chambon, P., and Dolle, P. 1996. AP-2.2, a novel gene related to AP-2, is expressed in the forebrain, limbs and face during mouse embryogenesis. Mech. Dev. 54: 83-94.

Davis, A.P., Witte, D.P., Hsieh-Li, H.M., Potter, S.S., and Capecchi, M.R. 1995. Absence of radius and ulna in mice lacking hoxa-11 and hoxd-11. Nature 375: 791-795.

de Maximy, A.A., Nakatake, Y., Moncada, S., Itoh, N., Thiery, J.P., and Bellusci, S. 1999. Cloning and expression pattern of a mouse homologue of Drosophila sprouty in the mouse embryo. Mech. Dev. 81: 213-216.

Dolle, P., Izpisua-Belmonte, J.C., Falkenstein, H., Renucci, A., and Duboule, D. 1989. Coordinate expression of the murine Hox-5 complex homoeobox-containing genes during limb pattern formation. Nature 342: 767-772.

Dolle, P., Ruberte, E., Leroy, P., Morriss-Kay, G., and Chambon, P. 1990. Retinoic acid receptors and cellular retinoid binding proteins. I. A systematic study of their differential pattern of transcription during mouse organogenesis. Development 110: 1133-1151.

Dudley, A.T., Ros, M.A., and Tabin, C.J. 2002. A re-examination of proximodistal patterning during vertebrate limb development. Nature 418: 539-544.

Eldredge, N. and Gould, S.J. 1972. Punctuated equilibria: An alternative to phyletic gradualism. In Models in paleobiology (ed. T.J.M. Schopf), pp. 82-115. Freeman, Cooper and Co., San Francisco.

Fallon, J.F., Lopez, A., Ros, M.A., Savage, M.V., Olwin, B.B., and Simandl, B.K. 1994. FGF-2 apical ectodermal ridge growth signal for chick limb development. Science 2641: 104-107.

Globus, M. and Vethaman-Globus, S. 1976. An in vitro analogue of early chick limb bud outgrowth. Differentiation 6: 91-96.

Goff, R.A. 1962. The relation of developmental status of limb formation to X-radiation sensitivity in chick embryos. I. Gross study. J. Exp. Zool. 151: 177-200.

Hashimoto, K., Yokouchi, Y., Yamamoto, M., and Kuroiwa, A. 1999. Distinct signaling molecules control Hoxa-11 and Hoxa-13 expression in the muscle precursor and mesenchyme of the chick limb bud. Development 126: 2771-2783.

Kardon, G. 1998. Muscle and tendon morphogenesis in the avian hind limb. Development 125: 4019-4032.

Koyama, E., Nohno, T., Myokai, F., Kuroiwa, A., Ide, H., Taniguchi, S., Saito, T., Nishijima, K., and Noji, S. 1993. Cooperative activation of Chox- 4 homeobox genes by factors from the polarizing region and the apical ridge in chick limb morphogenesis. Prog. Clin. Biol. Res. 383A: 51-60.

Laufer, E., Nelson, C.E., Johnson, R.L., Morgan, B.A., and Tabin, C. 1994. Sonic hedgehog and Fgf-4 act through a signaling cascade and feedback loop to integrate growth and patterning of the developing limb bud. Cell 79: 993-1003.

Lewandoski, M., Sun, X., and Martin, G.R. 2000. Fgf8 signalling from the AER is essential for normal limb development. Nat. Genet. 26: 460-463.

Mariani, F.V. and Martin, G.R. 2003. Deciphering skeletal patterning: Clues from the limb. Nature 423: 319-325.

Martin, G.R. 1998. The roles of FGFs in the early development of vertebrate limbs. Genes \& Dev. 12: 1571-1586.

Mercader, N., Leonardo, E., Piedra, M.E., Martinez-A, C., Ros, M.A., and Torres, M. 2000. Opposing RA and FGF signals control proximodistal vertebrate limb development through regulation of Meis genes. Development 127: 3961-3970.

Minowada, G., Jarvis, L.A., Chi, C.L., Neubuser, A., Sun, X., Hacohen, N., Krasnow, M.A., and Martin, G.R. 1999. Vertebrate Sprouty genes are induced by FGF signaling and can cause chondrodysplasia when overexpressed. Development 126: $4465-4475$. 
Moens, C.B. and Selleri, L. 2006. Hox cofactors in vertebrate development. Dev. Biol. 291: 193-206.

Nelson, C.E., Morgan, B.A., Burke, A.C., Laufer, E., DiMambro, E., Murtaugh, L.C., Gonzales, E., Tessarollo, L., Parada, L.F., and Tabin, C. 1996. Analysis of Hox gene expression in the chick limb bud. Development 122: 1449-1466.

Niswander, I., Tickle, C., Vogel, A., Booth, I., and Martin, G.R. 1993. FGF-4 replaces the apical ectodermal ridge and directs outgrowth and patterning of the limb. Cell 75: 579-587.

Ohuchi, H., Nakagawa, T., Yamamoto, A., Araga, A., Ohata, T., Ishimaru, Y., Yoshioka, H., Kuwana, T., Nohno, T., Yamasaki, M., et al. 1997. The mesenchymal factor, FGF10, initiates and maintains the outgrowth of the chick limb bud through interaction with FGF8, an apical ectodermal factor. Development 124: 2235-2244.

Pan, Y., Liu, Z., and Kopan, R. 2005. Notch1 and 2 cooperate in limb ectoderm to receive an early Jagged2 signal regulating interdigital apoptosis. Dev. Biol. 28: 472-482.

Pascoal, S., Carvalho, C.R., Rodriguez-Leon, J., Delphini, M.C., Duprez, D., Thorsteinsdottir, S., and Palmeirim, I. 2007. A molecular clock operates during chick autopod proximo-distal outgrowth. J. Mol. Biol. 368: 303-309.

Pinot, M. 1970. Developpement de l'ébauche des members après traitement à l'ypérite azotée, irradiation aux rayons $\mathrm{X}$ et culture in vitro. Etude comparative chez l'embryon de poulet. Annal. Embryol. Morphog. 3: 215-234.

Ros, M.A., Lyons, G., Kosher, R.A., Upholt, W.B., Coelho, C.N., and Fallon, J.F. 1992. Apical ridge dependent and independent mesodermal domains of GHox-7 and GHox-8 expression in chick limb buds. Development 116: 811-818.

Rowe, D.A. and Fallon, J.F. 1982. The proximodistal determination of skeletal parts in the developing chick leg. J. Embryol. Exp. Morphol. 68: 1-7.

Rowe, D.A., Cairns, J.M., and Fallon, J.F. 1982. Spatial and temporal patterns of cell death in limb bud mesoderm after apical ectodermal ridge removal. Dev. Biol. 93: 83-91.

Rubin, L. and Saunders, J.W. 1972. Ectodermal-mesodermal interactions in the growth of limb buds in the chick embryo: Constancy and temporal limits of ectodermal induction. Dev. Biol. 28: 94-112.

Sato, K., Koizumi, Y., Takahashi, M., Kuroiwa, A., and Tamura, K. 2007. Specification of cell fate along the proximal-distal axis in the developing chick limb bud. Development 134: $1397-1406$

Saunders, J.W. 1948. The proximo-distal sequence of origin of the parts of the chick wing and the role of the ectoderm. $J$. Exp. Zool. 282: 363-403.

Shen, H., Wilke, T., Ashique, A.M., Narvey, M., Zerucha, T., Savino, E., Williams, T., and Richman, J.M. 1997. Chicken transcription factor AP-2: Cloning, expression and its role in outgrowth of facial prominences and limb buds. Dev. Biol. 188: $248-266$.

Smith, S.M. and Eichele, G. 1991. Temporal and regional differences in the expression pattern of distinct retinoic acid receptor- $\beta$ transcripts in the chick embryo. Development 111: 245-252.

Sordino, P., van der Hoeven, F., and Duboule, D. 1995. Hox gene expression in teleost fins and the origin of vertebrate digits. Nature 375: 678-681.

Summerbell, D. 1974. A quantitative analysis of the effect of excision of the AER from the chick limb bud. J. Embryol. Exp. Morpho.1 32: 651-660.

Summerbell, D. 1978. Normal and experimental variations in proportions of skeleton of chick embryo wing. Nature 274: $472-473$.

Summerbell, D., Lewis, J.H., and Wolpert, L. 1973. Positional information in chick limb morphogenesis. Nature 244: 492496.

Sun, X., Lewandoski, M., Meyers, E.N., Liu, Y.H., Maxson Jr., R.E., and Martin, G.R. 2000. Conditional inactivation of Fgf4 reveals complexity of signalling during limb bud development. Nat. Genet. 25: 83-86.

Sun, X., Mariani, F.V., and Martin, G.R. 2002. Functions of FGF signalling from the apical ectodermal ridge in limb development. Nature 418: 501-508.

Vargesson, N., Kostakopoulou, K., Drossopoulou, G., Papageorgiou, S., and Tickle, C. 2001. Characterisation of hoxa gene expression in the chick limb bud in response to FGF. Dev. Dyn. 220: 87-90.

Vogel, A., Roberts-Clarke, D., and Niswander, L. 1995. Effect of FGF on gene expression in chick limb bud cells in vivo and in vitro. Dev. Biol. 171: 507-520.

Wellik, D.M. and Capecchi, M.R. 2003. Hox10 and Hox11 genes are required to globally pattern the mammalian skeleton. Science 301: 363-367.

Wolpert, L. 2002. Limb patterning: Reports of model's death exaggerated. Curr. Biol. doi: 10.1016/S0960-9822/02/01137-5.

Wolpert, L., Tickle, C., and Sampford, M. 1979. The effect of cell killing by X-irradiation on pattern formation in the chick limb. J. Embryol. Exp. Morphol. 50: 175-193.

Yokouchi, Y., Sasaki, H., and Kuroiwa, A. 1991. Homeobox gene expression correlated with the bifurcation process of limb cartilage development. Nature 353: 443-445.

Yokouchi, Y., Nakazato, S., Yamamoto, M., Goto, Y., Kameda, T., Iba, H., and Kuroiwa, A. 1995. Misexpression of Hoxa-13 induces cartilage homeotic transformation and changes cell adhesiveness in chick limb buds. Genes \& Dev. 9: 25092522.

Zwilling, E. 1959. Interaction between ectoderm and mesoderm in duck-chicken limb bud chimaeras. J. Exp. Zool. 142: 521532 . 


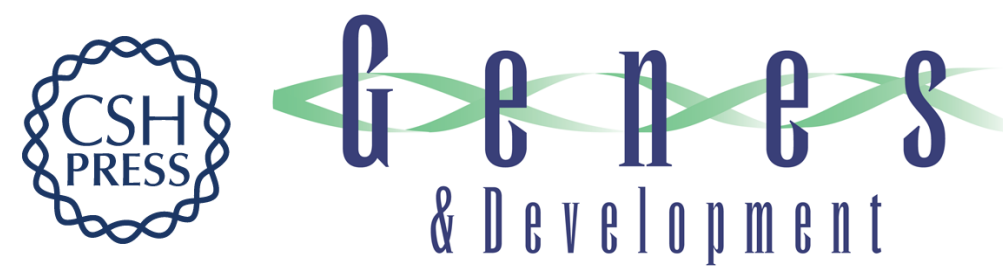

\section{Rethinking the proximodistal axis of the vertebrate limb in the molecular era}

Cliff Tabin and Lewis Wolpert

Genes Dev. 2007, 21:

Access the most recent version at doi:10.1101/gad.1547407

References This article cites 52 articles, 14 of which can be accessed free at: http://genesdev.cshlp.org/content/21/12/1433.full.html\#ref-list-1

License

Email Alerting

Receive free email alerts when new articles cite this article - sign up in the box at the top Service right corner of the article or click here.

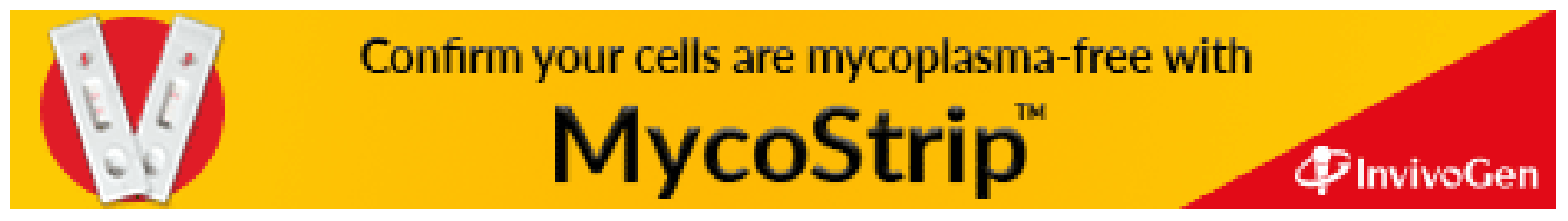

